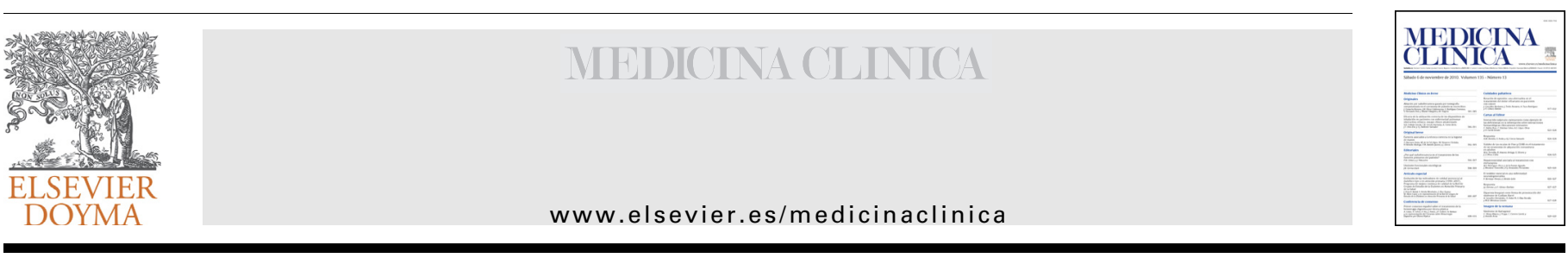

Diagnóstico y tratamiento

\title{
Síndrome de boca ardiente: claves diagnósticas y terapéuticas
}

\author{
Burning mouth syndrome: Diagnostic and therapeutic keys \\ Eduardo Chimenos-Küstner*, Cristina Arcos-Guerra y Maria Sueli Marques-Soares \\ Facultad de Odontología, Universidad de Barcelona, L'Hospitalet de Llobregat, Barcelona, España
}

INFOR M ACIÓN DEL ARTÍCULO

Historia del artículo:

Recibido el 19 de julio de 2013

Aceptado el 12 de septiembre de 2013

On-line el $\mathrm{xxx}$

\section{Introducción}

El síndrome de boca ardiente (SBA) se conoce también como boca escaldada, ardor bucal, glosodinia, glosopirosis, estomatodinia, estomatopirosis o disestesia oral. Se caracteriza por dolor o sensación de ardor, escozor o picazón, a veces aspereza, sobre todo en la lengua, paladar duro y labio inferior, en ausencia de datos clínicos o de laboratorio que justifiquen estos síntomas. Puede acompañarse de disgeusia (trastornos del gusto) y de xerostomía (boca seca), constituyendo la tríada clásica. Su etiología, poco precisa, es multifactorial. La evolución del cuadro tiende a ser crónica, alternando períodos de exacerbación y mejoría durante meses o años. Es más frecuente en mujeres perimenopáusicas o posmenopáusicas. Los episodios de SBA aparecen de forma espontánea y presentan un abanico variable en la gravedad de los síntomas. Mientras algunos pacientes refieren malestar leve o moderado, otros manifiestan dolor insoportable. Los síntomas suelen ser menos intensos por la mañana, empeorando a lo largo del día (hacia el atardecer), y desaparecen durante el sueño nocturno. A pesar de no acompañarse de cambios orgánicos evidentes y de no presentar riesgos para la salud, este síndrome puede reducir significativamente la calidad de vida de los pacientes. Es frecuente su consulta recurrente a profesionales de diversas especialidades (Atención Primaria, Otorrinolaringología, Dermatología, Neurología, Psiquiatría, Odontología, Acupuntura, Homeopatía, etc.), con malos resultados ${ }^{1-37}$.

En este trabajo se expone una revisión actualizada del tema y se proponen claves diagnósticas y terapéuticas estratégicas que pueden ayudar a combatir con mayor eficacia este cuadro clínico.

\footnotetext{
* Autor para correspondencia.

Correos electrónicos: echimenos@ub.edu, eduardochimenoskustner@gmail.com (E. Chimenos-Küstner).
}

\section{Epidemiología}

La verdadera prevalencia del SBA es difícil de establecer debido a la falta de criterios diagnósticos rigurosos ${ }^{4}$. No obstante, en función de los estudios disponibles, esta prevalencia varía entre el 0,7 y el $15 \%^{5}$. Afecta a mujeres en una proporción que varía entre el 3:1 y el 7:1 respecto a los varones. Esta diferencia entre sexos podría ser explicada en parte por factores biológicos, psicológicos y socioculturales, si bien tales factores no están bien definidos. Es rara su presencia en pacientes menores de 30 años, y no se ha descrito nunca en niños o adolescentes ${ }^{6}$. Es más frecuente en mujeres perimenopáusicas o posmenopáusicas, que a menudo sufren ansiedad o depresión ${ }^{15,27}$.

\section{Etiopatogenia}

La etiología y la patogenia del SBA no están claras. Los numerosos factores relacionados con su etiopatogenia se han dividido en locales, sistémicos y psicológicos ${ }^{6,11-13}$. Sin embargo, en algunos pacientes es idiopático ${ }^{4,13,27}$.

\section{Factores locales}

Pueden ser tanto físicos, como químicos, o biológicos (bacterias, hongos), y son capaces de desencadenar los síntomas, por su efecto irritante directo sobre la mucosa oral ${ }^{6}$. El factor mecánico, como el roce de dientes o el uso prolongado de prótesis removibles, puede producir microtraumas o eritema local. Ciertas reacciones alérgicas locales, debidas a monómeros residuales u otros alérgenos, también pueden desatar el cuadro ${ }^{6,16,25}$. Dado que se han descrito reacciones alérgicas epicutáneas positivas en pacientes con ardor bucal sin lesiones orales evidentes, que se han recuperado al retirar el alérgeno, cabría plantearse el diagnóstico diferencial con mucositis alérgica. Estos hallazgos no justificarían un dolor intermitente $e^{6}$. Las alergias a los materiales de las prótesis dentales son raras y solo se pueden confirmar con pruebas de alergia en 
correspondencia con los hallazgos clínicos ${ }^{3}$. El SBA puede asociarse a infecciones orales producidas por diversos microorganismos. La infección por Candida albicans ha sido considerada uno de los factores más frecuentes en la producción de ardor bucal y de SBA ${ }^{6}$. Otros microorganismos citados por diversos autores como cofactores de ardor bucal son Enterobacter spp., Klebsiella spp., Staphylococcus aureus y Helicobacter pylori ${ }^{3,6,14}$. La xerostomía es un síntoma (y signo) frecuente en pacientes con SBA, cuya prevalencia varía entre el 34 y el $39 \%^{6}$. En ocasiones el ardor bucal se relaciona con variedades no patológicas de la lengua, como glositis migratoria benigna (lengua geográfica) y lengua fisurada ${ }^{3}$. No es raro que el paciente refiera el antecedente de un tratamiento odontológico reciente ${ }^{13}$.

\section{Factores sistémicos}

\section{Se destacan los siguientes:}

- Deficiencias de vitaminas y minerales (vitaminas $\mathrm{C}, \mathrm{B}_{12}, \mathrm{~B}_{6}$, ácido fólico; hierro, cinc) $)^{4,6}$.

- Trastornos endocrinos (diabetes mal controlada, menopausia, tiroides) $)^{4,6,14,27}$.

- Enfermedades reumáticas: el síndrome de Sjögren, de patogenia autoinmunitaria, afecta más a mujeres de edades comprendidas entre los 40 y 60 años; cursa con xerostomía y puede asociarse a otros trastornos del tejido conectivo, como la esclerosis múltiple y la artritis reumatoide, así como al SBA. La fibromialgia (FM) es un síndrome doloroso crónico, diagnosticado por presencia de dolor en al menos 11 de los 18 puntos sensibles característicos de esta enfermedad, durante un mínimo de 3 meses. El SBA acompaña a un $32,8 \%$ de los pacientes con $\mathrm{FM}$, donde los síntomas orales descritos con mayor frecuencia incluyen xerostomía $(70,9 \%)$, dolor orofacial $(32,8 \%)$, disfunción de la articulación temporomandibular $(67,6 \%)$, disfagia $(37,3 \%)$ y disgeusia $(34,2 \%)^{3,6,18,19,27}$.

- Aumento de neuropéptidos: algunos de ellos (como la sustancia P y la neurocinina A) aumentan en saliva en pacientes con SBA. Parecen relacionarse con un sistema dopaminérgico ineficiente. El mejor conocimiento de la relación del SBA con otros síndromes de dolor crónico podría ayudar a mejorar su tratamiento $^{17}$.

- Farmacoterapia: se han descrito muchos casos de ardor bucal en pacientes que toman fármacos inhibidores de la enzima conversiva de la angiotensina. También medicamentos antirretrovirales, antibióticos (cefalosporinas, cloranfenicol, penicilina), gabapentina, antidepresivos tricíclicos y fármacos ansiolíticos se consideran causantes de estomatodinia ${ }^{3,18,33}$.

- Radioterapia: inicialmente puede provocar mucositis, con úlceras muy dolorosas. Más tarde se desarrolla atrofia permanente de la mucosa, con ardor en la lengua, boca seca y dificultad para deglutir. El compromiso de la función glandular salival depende de la dosis de radiación, que causa lesiones irreversibles a partir de $40 \mathrm{~Gy}^{3}$.

\section{Factores psicológicos}

Los factores psicológicos y trastornos psicosociales están presentes en más de un $50 \%$ de los casos. Muchos pacientes refieren altos niveles de ansiedad y depresión, alcanzando esta una prevalencia de hasta el $31 \%$. Se asocian también trastornos de la personalidad. Algunos autores concluyen que la ansiedad es el principal factor psicológico, siendo el obstáculo más difícil de superar $^{15}$. Los pacientes con SBA tienen tendencia a la somatización y otros síntomas psiquiátricos ${ }^{6,27}$. Un estudio mediante resonancia magnética reveló que pacientes con SBA presentaban un patrón específico cuantitativo y cualitativo de la activación cerebral, mostrando hipoactividad del mismo. Estos hallazgos evidencian que la dinámica cerebral podría estar dañada, disminuyendo el control inhibitorio de la experiencia sensorial, que justificaría la propiocepción intraoral de ardor bucal ${ }^{22}$.

\section{Aspectos fisiopatológicos}

Autores japoneses analizaron 2 marcadores asociados al sistema defensivo de la mucosa oral (MUC1 y TLR2) en pacientes con SBA, comparando con pacientes de liquen plano y controles. Se observó un aumento de MUC1 en pacientes con SBA, en comparación con los pacientes con liquen plano oral y controles, mientras que no hubo diferencias significativas en los valores de TLR2 entre los grupos. Los resultados evidencian que MUC1 desempeña un papel importante en el desarrollo y progresión del ardor bucal. El aumento de MUC1 podría resultar de la adaptación de las células de la mucosa a la irritación crónica constante ${ }^{20}$. Por otra parte, los valores de interleucinas (IL) 2 y 6 de la saliva son elevados en estos pacientes, correlacionándose con la gravedad de la enfermedad ${ }^{23}$. Las IL actúan como neuromoduladores o neurotransmisores potenciales en el procesamiento nociceptivo del dolor neuropático y de la hiperalgesia. IL-6 es una citocina neuropoyética, que podría desempeñar varios papeles en la proliferación de células gliales, en la supervivencia celular, en la regeneración axonal y en las actividades proinflamatorias. Teniendo en cuenta la base neuropática del SBA y las acciones neurológicas de IL-6, es interesante estudiar la relación entre ambas. Los resultados muestran que la IL-6 podría estar involucrada en la etiopatogenia neuropática del ardor bucal ${ }^{24}$.

\section{Clasificación}

Se han descrito 2 clasificaciones para el SBA. En la primera, se distinguen 2 categorías: primaria (esencial o idiopática), en la que la sensación de ardor de la mucosa oral y áreas periorales no se acompaña de hallazgos clínicos ni de laboratorio, y secundaria, en la que el escozor oral se produce como consecuencia de alteraciones clínicas, incluyendo lesiones en la mucosa oral, enfermedades sistémicas, procesos psicológicos y efectos adversos de ciertos medicamentos ${ }^{9,26}$. En la segunda, basada en las fluctuaciones diarias de los síntomas, se diferencian $3 \operatorname{tipos}^{6}$.

1) Caracterizado por un dolor progresivo: los pacientes se levantan sin dolor, que va aumentando a lo largo del día. Este tipo puede asociarse con enfermedades sistémicas, como deficiencias nutricionales.

2) Los síntomas son constantes a lo largo del día, y los pacientes tienen dificultades para dormir. Estos suelen presentar trastornos psicológicos asociados.

3) Los síntomas son intermitentes, con dolor y localizaciones atípicas. Parece que el contacto con alérgenos orales puede desempeñar un papel importante en la etiología de este grupo.

\section{Tratamiento}

El tratamiento del SBA se suele orientar hacia el manejo de los síntomas. Deben eliminarse los factores locales que aumentan la sensación de boca ardiente, como tabaco, alcohol, comidas picantes y bebidas ácidas que irriten la mucosa oral. Es necesario investigar la presencia de hábitos parafuncionales, corrientes galvánicas, irritación mecánica o alergia a componentes de la dentadura. La eliminación de estos factores suele traducirse en una mejoría clínica ${ }^{4,18,27,36,37 . ~}$ 


\section{Farmacoterapia}

Aunque la etiopatogenia del SBA no es bien conocida, problemas como la depresión y la ansiedad parecen desempeñar un papel importante en la modulación de la percepción del dolor. Por ello es frecuente orientar el tratamiento hacia fármacos antidepresivos en estos pacientes. Por otra parte, la identificación de alteraciones sensoriales evidencia una base biológica relacionada con alteraciones del sistema nervioso central y del sistema nervioso periférico. Esta teoría parece reforzar la acción beneficiosa del clonacepam. Los cambios en la tolerancia al calor, la disgeusia y el aumento de la excitabilidad del reflejo palpebral que tienden a presentar estos pacientes están relacionados con la disfunción del sistema dopaminérgico a nivel central. Esta disfunción justificaría la administración sistémica de fármacos antiepilépticos como gabapentina y clonacepam, que actúan sobre el sistema gabaérgico. Por otra parte, se ha visto que el SBA presenta una serie de características comunes con otros síndromes dolorosos crónicos, justificando la administración de fármacos como capsaicina y bencidaminas $^{30}$. La amisulprida (antagonista selectivo de la dopamina) parece haber dado buenos resultados en el tratamiento de SBA a corto plazo, si bien, según los autores, conviene ampliar el estudio a largo plazo ${ }^{11}$. También se han publicado casos de mejoría empleando pramipexol, fármaco agonista de la dopamina usado comúnmente para la enfermedad de Parkinson ${ }^{8}$.

\section{Otros métodos}

Autores japoneses relacionan el SBA con la función del sistema nervioso autónomo. Irradiando con infrarrojos el núcleo estrellado observaron una mejoría semiológica en pacientes afectados ${ }^{21}$. También se ha propuesto el láser de baja intensidad, aplicado en la zona referida por el paciente, con aparentes resultados beneficiosos $^{7}$. La acupuntura se considera un método alternativo para el tratamiento del dolor, que algunos autores proponen para el SBA ${ }^{31}$. Aloe vera, en combinación con un protector lingual, contribuyen a minimizar los traumas repetitivos de la mucosa oral ${ }^{12}$. El tratamiento cognitivo-conductual ayuda a reducir la ansiedad, con lo que disminuye también significativamente el dolor en estos pacientes $^{32}$. Para algunos autores, una pequeña proporción de casos presenta remisión espontánea durante los 5 primeros años de tratamiento; en un $42 \%$ mejoran los síntomas significativamente, y esta mejora alcanza un $60 \%$ si se asocian clonacepam y psicoterapia ${ }^{5}$. En definitiva, aunque se han encontrado tratamientos eficaces para algunos casos, se sigue buscando un procedimiento que resulte eficaz en la mayoría de los $\operatorname{casos}^{30}$.

\section{Discusión}

Un paciente típico de SBA es una mujer perimenopáusica o posmenopáusica, con compromiso psicológico, como ansiedad o depresión, y varias comorbilidades médicas ${ }^{2,27}$. La tríada clásica es: ardor bucal continuo o intermitente, disgeusia y xerostomía. No suele observarse afectación destacable en la mucosa oral y la duración acostumbra ser de al menos 4-6 meses (a menudo, varios años). La presentación clínica en muchos pacientes es monosintomática (solo dolor), o bien oligosintomática (dolor y disgeusia o xerostomía) 1,27,28. El papel del gusto en el SBA es una cuestión compleja. La punta de la lengua es la que suele afectarse más, y el dolor se acompaña a menudo de alteraciones del gusto. Cabe atribuirlo, en parte, a una mayor densidad de papilas fungiformes en estos pacientes ${ }^{29}$, pero también intervienen factores hormonales, particularmente en relación con la función tiroidea ${ }^{10}$, así como el tratamiento farmacológico que reciben ${ }^{18,33}$. En el diagnóstico diferencial es necesario distinguir y excluir otras causas de ardor bucal, tras cuyo tratamiento el paciente recupera su estado de bienestar. Algunos ejemplos son estomatitis infecciosas (por hongos, bacterias, virus), de patogenia inmunitaria (gingivitis descamativas crónicas asociadas a reacciones liquenoides o penfigoides), o de origen traumático físico o químico, entre otras ${ }^{2}$. Esos casos escaparían al diagnóstico de SBA.

Un síndrome es un conjunto de signos (objetivos: evidentes, identificables por el facultativo) y de síntomas (subjetivos: relatados por el paciente, no identificables por el profesional). Como tal, el SBA reúne una serie de características complejas, que se resumen en la figura 1 . Consideramos que los pacientes presentan siempre hábitos parafuncionales, si bien estos no son fáciles de reconocer en todos los casos. Según ese esquema, y con el apoyo de la evidencia científica, se desglosan a continuación algunas claves diagnósticas y terapéuticas recomendables, basadas en la experiencia adquirida durante más 30 años de ejercicio profesional, resumidas en la figura 2.

\section{Claves diagnósticas del síndrome de boca ardiente}

El paciente refiere dolor urente, picor o sensación de aspereza o arenosa en la lengua, los labios o cualquier otra localización mucosa. En ocasiones describe tan solo una sensación de boca seca (xerostomía), que no discrimina del ardor. La alteración de la percepción de sabores (disgeusia) es también frecuente. Todo ello puede haberse desarrollado a partir de un tratamiento odontológico (factor local) más o menos reciente, que le ha comportado malestar. Si además, como suele ocurrir, se trata de un paciente polimedicado (factor sistémico), lo más común es que los fármacos tiendan a provocar sequedad de boca y cierto grado de disfagia, desencadenantes de parafunción deglutoria. La deshidratación y dietas inadecuadas agravan el cuadro. Trastornos como ansiedad o depresión (factor psicológico), resultantes de una situación mantenida de estrés crónico por problemas familiares, laborales o de cualquier otra índole, propenden al paciente a desarrollar ciertos hábitos estereotipados repetitivos o tics (parafunción), que cierran el círculo del SBA. Son evidentes a veces signos de apretamiento o rechinamiento dentario, por las facetas de desgaste. La lengua suele presentar improntas de los dientes o áreas mordisqueadas, la punta enrojecida, con las papilas fungiformes eritematosas e hipersensibles. En las mejillas (mucosa yugal) son frecuentes las lesiones leucoedematosas provocadas por succión y pellizcamiento, al igual que en los labios, sobre todo el inferior (lesiones facticias). Estos signos clínicos pueden tener repercusiones tangibles sobre el complejo articular temporomandibular (fibrocartílago, músculos, cápsula articular), que también debe

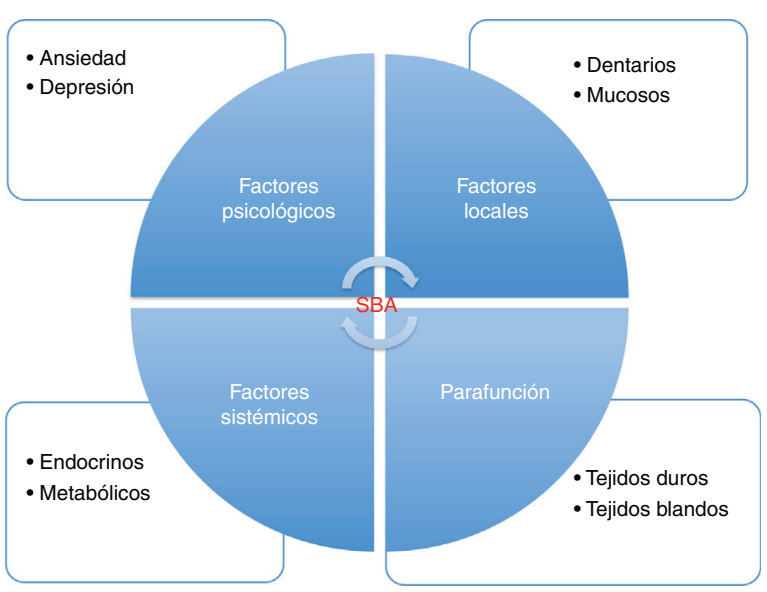

Figura 1. Factores implicados en el desarrollo del síndrome de boca ardiente. SBA: síndrome de boca ardiente. 


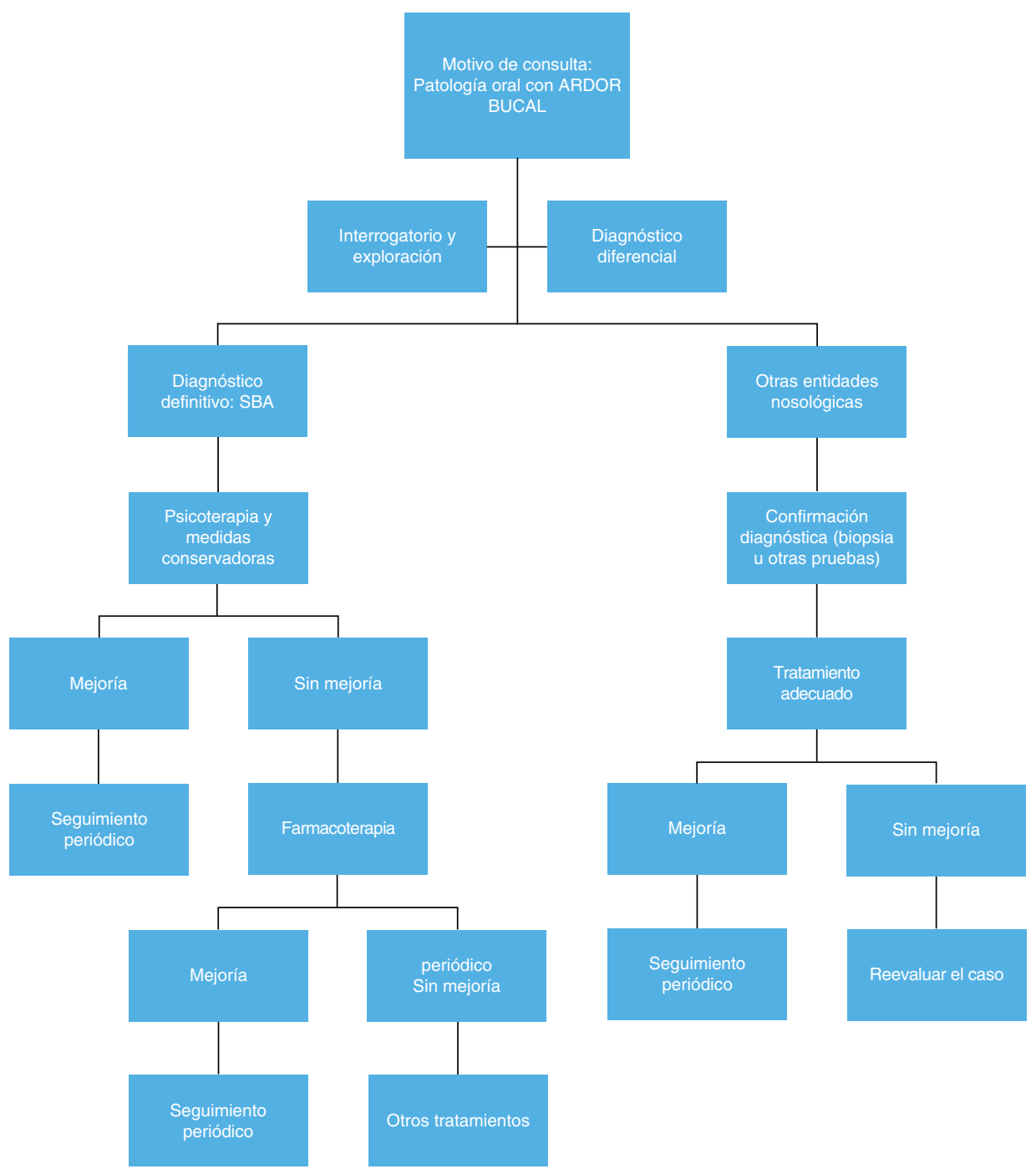

Figura 2. Propuesta de actuación ante el ardor bucal.

SBA: síndrome de boca ardiente.

ser explorado. En el transcurso del interrogatorio no es difícil observar al paciente tragando saliva de forma reiterativa o realizar diversos movimientos compulsivos, que implican a diversas estructuras orofaciales. Todos estos hábitos parafuncionales son, a nuestro juicio, la clave principal del SBA. La fricción repetitiva de diversas zonas de la mucosa oral (lengua, labios) con las estructuras más duras próximas (mucosa masticatoria palatina, dientes, prótesis) provoca pequeños focos inflamatorios (de ahí la alteración observada de mediadores de la inflamación como IL-2 y IL-6), que a su vez propician la aparición de neuropéptidos (sustancia P, neurocininas) ${ }^{17,20,23,24}$. Teniendo en cuenta que la mucosa oral está ricamente inervada por terminaciones sensitivas especializadas (papilas gustativas), es fácil comprender que el proceso inflamatorio desencadenado por el factor mecánico altere la percepción de los sabores ${ }^{29}$. Sin embargo, todo ello sucede de una manera sutil. El paciente no suele mantener su parafunción las 24 horas del día, todos los días. En función de su estado emocional, los tics van o vienen, de ahí la intermitencia y fluctuación del dolor, que ha llevado a las distintas clasificaciones ${ }^{6}$. Para cuantificar las molestias, es útil recurrir a una escala analógica del dolor (de 0 a 10 , siendo $0=$ molestia nula y $10=$ máxima intensidad). Establecido el diagnóstico, debe plantearse una estrategia terapéutica.

\section{Claves terapéuticas del síndrome de boca ardiente}

Intuitivamente, algunos pacientes mitigan su malestar masticando chicle o caramelos, o comiendo alimentos suaves o fríos, que estimulan la secreción salival. Es importante el tiempo de evolución del SBA, cuando el paciente acude a consultar. Recién instaurado el cuadro, el tratamiento conservador tiende a ser más eficaz. Es frecuente encontrar malposiciones dentarias, obturaciones o implantes recién colocados, prótesis fijas o removibles, entre los factores locales a revisar. Si con el dedo el explorador percibe irregularidades, se comprende que las terminaciones nerviosas sensitivas de la mucosa oral (lengua, labios) discriminan aún más y eso puede constituir un estímulo (doloroso y placentero a la vez) para desarrollar y mantener los tics parafuncionales. El objetivo será eliminar o atenuar la agresión local, en la medida de lo posible, mediante fresado y pulido, aplicando productos lubricantes de cierta densidad y propiedades adecuadas, o bien con aparatos (férulas, placas de descarga, protectores plásticos); todos ellos pueden combinarse. También se deben evitar los hábitos tóxicos (tabaco, alcohol), que contribuyen a resecar e irritar la mucosa oral ${ }^{27}$. Factores sistémicos como infecciones subagudas o crónicas (en particular candidiasis, asociadas a hábitos alimentarios inadecuados y a deshidratación -absoluta o relativa-) requerirán 
un estudio analítico. No es raro descubrir alteraciones en el metabolismo del hierro (como, por ejemplo, defectos en la saturación de transferrina, ferritina o sideremia bajas), que hacen recomendable investigar su origen e introducir una compensación alimentaria (más que farmacológica). También pueden estar alteradas las funciones tiroidea, pancreática o corticosuprarrenal, requiriéndose atención facultativa especializada. En muchos casos conviene revisar la medicación que toma el paciente, incitándole a que, en consenso con su médico de cabecera, reduzca dosis o elimine fármacos ${ }^{18,33}$. Los factores psicológicos son difíciles de controlar $^{32}$. De hecho, a menudo estos pacientes ya están recibiendo tratamiento farmacológico ansiolítico o antidepresivo, sin que ello haya evitado el desarrollo de la parafunción, como parte fundamental del SBA que presentan. Por tanto, lo más recomendable no es abundar en la administración de fármacos, sino orientar al paciente en otro sentido. Se debe recomendar la ingesta de infusiones relajantes y, por el contrario, evitar bebidas excitantes. En función de la edad y las capacidades del paciente, es recomendable que practique ejercicio físico aeróbico de forma $\operatorname{regular}^{38}$, mejor si lo hace fuera de su entorno habitual. Con ello aumentan las posibilidades de evadirse, siquiera temporalmente, de problemas cotidianos, y de relacionarse con otras personas. Ello contribuye a una mayor estabilidad emocional y ayuda a regular los mediadores de la inflamación y del dolor produciendo endorfinas. En caso de que la combinación de estas medidas no surtan el efecto esperado, se puede recurrir a tratamiento farmacológico. No obstante, ninguno de los tratamientos propuestos es definitivo ni universal, como se desprende de la revisión bibliográfica $^{30}$. Es fundamental prestarle al paciente una atención especial, desde la primera visita, ya que la psicoterapia constituye un pilar ineludible de su tratamiento $0^{5,32}$. Como conclusión provisional, los diversos estudios pretenden demostrar la eficacia de cada uno de los fármacos empleados, aunque los resultados apuntan a que la capsaicina y el clonacepam por vía oral deben descartarse debido a sus efectos secundarios; la gabapentina es ineficaz y las bencidaminas no son más efectivas que los placebos. Con la amisulprida se han demostrado mejoras, pero no suficientes. Por lo tanto, el clonacepam tópico podría ser la mejor opción, mejorando los síntomas en la mitad de los pacientes ${ }^{5,29,37}$

En definitiva, para un buen diagnóstico y tratamiento del SBA se requiere atender de forma equipotencial todos los factores referidos, incidiendo en cada caso en los más prevalentes. En nuestra experiencia, la eficacia y el éxito del tratamiento instaurado dependerán más de esta consideración global y de la buena relación entre el paciente y el profesional, que del tipo de tratamiento elegido.

\section{Conflicto de intereses}

Los autores declaran no tener ningún conflicto de intereses.

\section{Bibliografía}

1. Gurvits GE, Tan A. Burning mouth syndrome. World J Gastroenterol. 2013; 19:665-72

2. Zakrzewska JM. Multi-dimensionality of chronic pain of the oral cavity and face J Headache Pain. 2013;14:37.

3. Cerchiari DF, de Moricz RD, Sanjar FA, Rapoport PB, Moretti G, Guerra MM. Burning mouth syndrome: Etiology. Braz J Otorhinolaryngol. 2006;72:419-24

4. Spanemberg JC, Cherubini K, Zancanaro de Figueiredo MA, Soares-Yurgel L Gonçalves-Salum F. Aetiology and therapeutics of burning mouth syndrome: An update. Gerodontology. 2012;29:84-9.

5. Rodríguez de Rivera-Campillo E, López-López J. Evaluation of the response to treatment and clinical evolution in patients with burning mouth syndrome. Med Oral Patol Oral Cir Bucal. 2013:18:e403-10.

6. López-Jornet P, Camacho-Alonso F, Andújar-Mateos P, Sánchez-Siles M, GómezGarcía F. Burning mouth syndrome: Update. Med Oral Patol Oral Cir Bucal. 2010;15:e562-8.
7. Romeo U, del Vecchio A, Capocci M, Maggiore C, Ripari M. The low level laser therapy in the management of neurological burning mouth syndrome. A pilot study. Ann Stomatol (Roma). 2010;1:14-8.

8. Stuginski-Barbosa J, Rodrigues GG, Bigal ME, Speciali JG. Burning mouth syndrome responsive to pramipexol. J Headache Pain. 2008;9:43-5.

9. Nasri C, Teixeira MJ, Okada M, Formigoni G, Heir G, de Siqueira JT. Burning mouth complaints: Clinical characteristics of a Brazilian sample. Clinics (Sao Paulo). 2007;62:561-6.

10. Felice F, Gombos F, Esposito V, Nunziata M, Scully C. Burning mouth syndrome (BMS): Evaluation of thyroid and taste. Med Oral Patol Oral Cir Bucal. 2006;11:E22-5.

11. Rodriguez-Cerdeira C, Sanchez-Blanco E. Treatment of burning mouth syndrome with amisulpride. J Clin Med Res. 2012;4:167-71.

12. López-Jornet P, Camacho-Alonso F, Molino-Pagán D. Prospective, randomized, double-blind, clinical evaluation of Aloe vera Barbadensis, applied in combination with a tongue protector to treat burning mouth syndrome. J Oral Pathol Med. 2013;42:295-301.

13. Komiyama O, Obara R, Uchida T, Nishimura H, Iida T, Okubo M, et al. Pain intensity and psychosocial characteristics of patients with burning mouth syndrome and trigeminal neuralgia. J Oral Sci. 2012;54:321-7.

14. Palacios-Sánchez MF, Jordana-Comín X, García-Sívoli CE. Burning mouth syndrome: A retrospective study of 140 cases in a sample of catalan population. Med Oral Patol Oral Cir Bucal. 2005;10:388-93.

15. Bakhtiari S, Khalighi HR, Azimi S, Alavi K, Valoogerdi H, Namazi Z. Correlation between burning mouth syndrome and anxiety in the elderly inmates of sanitaria in Tehran. J Dent Res Dent Clin Dent Prospects. 2010;4:37-41.

16. Saraceno R, Ruzzetti M, Lanti A, Marinacci M, Chimenti S. Burning mouth syndrome associated with palladium allergy. Eur J Dermatol. 2008;18:356.

17. Boras VV, Savage NW, Brailo V, Lukac J, Lukac M, Alajbeg IZ. Salivary and serum levels of substance $\mathrm{P}$, neurokinin $\mathrm{A}$ and calcitonin gene related peptide in burning mouth syndrome. Med Oral Patol Oral Cir Bucal. 2010;15:e427-31.

18. Marques-Soares MS, Chimenos-Küstner E, Subirá-Pifarrè C, Rodríguez de Rivera-Campillo ME, López-López J. Asociación de síndrome de boca ardiente con xerostomía y medicamentos. Med Oral Patol Oral Cir Bucal. 2005;10:301-8.

19. Ferré-Corominas J, Chimenos-Küstner E, López-López J. Consideraciones odontológicas en la fibromialgia. Med Clin (Barc). 2011;137:27-9.

20. Kho HS, Chang JY, Kim YY, Kim Y. MUC1 and toll-like receptor-2 expression in burning mouth syndrome and oral lichen planus. Arch Oral Biol. 2013;58:837-42.

21. Momota Y, Takano H, Kani K, Matsumoto F, Motegi K, Aota K, et al. Frequency analysis of heart rate variability: A useful assessment tool of linearly polarized near-infrared irradiation to stellate ganglion area for burning mouth syndrome. Pain Med. 2013;14:351-7.

22. Fedele S, Fricchione G, Porter SR, Mignogna MD. Burning mouth syndrome (stomatodynia). Q J Med. 2007:100:527-30.

23. Simcić D, Pezelj-Ribarić S, Grzić R, Horvat J, Brumini G, Muhvić-Urek M. Detection of salivary interleukin 2 and interleukin 6 in patients with burning mouth syndrome. Mediators Inflamm. 2006;1-4. ID54632.

24. Chen Q, Xia J, Lin M, Zhou H, Li B. Serum interleukin-6 in patients with burning mouth syndrome and relationship with depression and perceived pain. Mediators Inflamm. 2007;1-4. ID45327.

25. Schepis C, Lentini M, Siragusa M, Palazzo R. Burning mouth syndrome possibly linked with an amalgam tattoo: Clinical and ultrastructural evidence. Eur J Dermatol. 2008;18:723.

26. Klasser GD, Epstein JB. Oral burning and burning mouth syndrome. J Am Dent Assoc. 2012:143:1317-9.

27. Chimenos-Küstner E. El síndrome de boca ardiente en la práctica clínica. JANO. 2008;31-4.

28. Park HO, Ha JH, Jin MU, Kim YK, Kim SK. Diagnostic challenges of nonodontogenic toothache. Restor Dent Endod. 2012;37:170-4, http://dx.doi.org/ 10.5395/rde.2012.37.3.170.

29. Camacho-Alonso F, López-Jornet P, Molino-Pagán D. Fungiform papillae density in patients with burning mouth syndrome and xerostomia. Med Oral Patol Oral Cir Bucal. 2012;17:e362-6.

30. Mínguez-Serra MP, Salort-Llorca C, Silvestre-Donat FJ. Pharmacological treatment of burning mouth syndrome: A review and update. Med Oral Patol Oral Cir Bucal. 2007;12:E299-304

31. Sardella A, Lodi G, Tarozzi M, Varoni E, Franchini R, Carrassi A. Acupuncture and Burning Mouth Syndrome: A Pilot Study. Pain Pract. 2013;13:627-32, http:// dx.doi.org/10.1111/papr.12031.

32. Komiyama O, Nishimura H, Makiyama Y, Iida T, Obara R, Shinoda M, et al. Group cognitive-behavioral intervention for patients with burning mouth syndrome. J Oral Sci. 2013:55:17-22.

33. Chimenos E, Marques MS. Boca ardiente y saliva. Med Oral. 2002;7:244-53.

34. Perdomo-Lovera M, Chimenos-Küstner E. Síndrome de boca ardiente: actualización. Av Odontoestomatol. 2003;19:193-202.

35. Rodríguez de Rivera-Campillo ME, López-López J, Chimenos-Küstner E, SabaterRecolons MM. Estudio de una muestra de pacientes con síndrome de boca ardiente. Av Odontoestomatol. 2007;23:123-33.

36. Ros-Lluch N, Chimenos-Küstner E, López-López J, Rodríguez de Rivera-Campillo ME. Síndrome de ardor bucal: actualización diagnóstica y terapéutica. Av Odontoestomatol. 2008;24:109-17.

37. Rodríguez de Rivera Campillo E, López-López J, Chimenos-Küstner E. Response to topical clonazepam in patients with burning mouth syndrome: A clinical study. Bull Group Int Rech Sci Stomatol Odontol. 2010;49:19-29.

38. Jayakody K, Gunadasa S, Hosker C. Exercise for anxiety disorders: Systematic review. Br J Sports Med. En prensa 2013. 